\title{
Synthesis and Structural Pattern Recognition of 5-(2'-Alkoxycarbonyl- substituted phenoxy)furfural Derivatives
}

\author{
Chia-Lin CHANG \\ Research Institute of Biotechnology, HungKuang University; No. 34, Chungchie Rd., Shalu Township, Taichung Hsien \\ 43302, Taiwan (R.O.C.). Received October 3, 2008; accepted March 22, 2009; published online March 27, 2009
}

The ethyl 5-(2'-alkoxycarbonyl-substituted phenoxy)furan-2-carboxylates (1C-13C) showed good antiplatelet aggregation, ${ }^{1)}$ anti-allergic ${ }^{2)}$ and anti-inflammatory activities. ${ }^{2)} \mathrm{A}$ series of 5-(2'-alkoxycarbonyl-substituted phenoxy)furfurals $(1 \mathrm{~F}-13 \mathrm{~F})$ were prepared for comparing the above activities. The objectives of this research are the synthesis and structural pattern recognition of compounds $1 F-13 F$. The Silica Gel 60 column chromatography method was employed to separate and purify pure compounds $1 \mathrm{~F}-13 \mathrm{~F}$ by three solvent systems. For the structure elucidation of compounds $1 \mathrm{~F}-13 \mathrm{~F}$, four spectroscopic methods were used: electron impact mass (EI-MS), UV-VIS, IR and NMR spectrometers. With the help of spectrometers, investigations can be performed on spectroscopic data. A simple methodology for recognizing structural patterns was carried out with the aid of statistical analysis designed to establish the classification model of the structural skeleton in this research. It was found that compounds $1 \mathrm{C}-13 \mathrm{C}$ and $1 \mathrm{~F}-13 \mathrm{~F}$ have similar chemical profiles and are clustered into one group. The pattern plots revealed valuable information and showed good correlation between compounds $1 \mathrm{C}-13 \mathrm{C}$ and $1 \mathrm{~F}-13 \mathrm{~F}$. These findings correlate directly with the resulting spectroscopic data. These results with those obtained by EI-MS and NMR patterns give insight into a reliable pattern recognition for determining both $1 \mathrm{C}-13 \mathrm{C}$ and $1 \mathrm{~F}-13 \mathrm{~F}$ skeletons.

Key words $\quad 5$-(2'-alkoxycarbonyl-substituted phenoxy)furfural; structural pattern; ${ }^{1} \mathrm{H}-\mathrm{NMR} ;{ }^{13} \mathrm{C}-\mathrm{NMR}$; MS

Some active medicines with anti-platelet aggregation, antiallergy, and anti-inflammation properties are used on clinic at present. The research and development of these medicines aims to treat human diseases effectively. Anti-agglutinate medicines of blood platelet include ketanserin, ${ }^{3)}$ milrinone ${ }^{4)}$ and ticlopidine. ${ }^{5)}$ Ketanserin produced a decrease in ristocetin-induced platelet aggregation, fibrinogen and prolongation of the bleeding time. ${ }^{3)}$ Milrinone $(0.9 \mu \mathrm{M})$ inhibited thrombin-induced platelet aggregation. This inhibitory effect was mainly mediated by suppressing calcium release from the dense tubular system. ${ }^{4)}$ Ticlopidine with an active reduction in platelet aggregation was detected in response to adenosine diphosphate. ${ }^{5)}$ Anti-allergy medicines include antazoline, ${ }^{6}$ brompheniramine ${ }^{7)}$ and chlorpheniramine. ${ }^{8)} \mathrm{Be}-$ cause allergic conjunctivitis is one of the most frequent allergic diseases of the anterior eye segment, the benefit of using antazoline eye drops was clearly outweighed. ${ }^{6}$ ) Brompheniramine has been used in the treatment of allergic rhinitis and other disorders during the past 40 years. In particular, a single dose of brompheniramine produces prompt, long-lasting peripheral H1-blockade in children. ${ }^{7)}$ Histamine $\mathrm{H} 1$ antagonist chlorpheniramine blocks the ragweed-induced nasal congestion. ${ }^{8)}$ Anti-inflammation medicines include diclofenac, ${ }^{9)}$ ketoprofen $^{10)}$ and tolmetin. ${ }^{11)}$ However, the effect of indomethacin on prenatal ductal constriction is well known, and widely prescribed non-steroidal anti-inflammatory drugs such as diclofenac can have an adverse effect and are best avoided in the third trimester of pregnancy.9 ${ }^{9}$ Ketoprofen is effective and safe in treating acute painful conditions for one week. ${ }^{10)}$ Tolmetin is safe and effective treatment for arthritic rheumatic fever patients without carditis, and can be used particularly in patients who cannot tolerate aspirin. ${ }^{11)}$ Therefore, the research of the above medicinal effects is necessary for drug discoveries.

A lot of structural pattern recognition has been established using spectroscopic data of electron impact (EI)-MS and NMR. ${ }^{12-17)}$ Structural pattern recognition could help researchers in interpretation and prediction of chemical structures. An unknown spectrum will be identified by the structural pattern, and both the research and structure elucidation will be easy. In my previous research of compounds 1C 13C on novel anti-platelet aggregation, ${ }^{1)}$ anti-allergic ${ }^{2)}$ and anti-inflammatory agents, ${ }^{2)}$ a series of compounds $\mathbf{1 F}-\mathbf{1 3 F}$, which are targeted as bioisosteres of the above compounds, were synthesized. In this study, these analogues $\mathbf{1 F}-\mathbf{1 3 F}$ with another substituent on the 2'-alkoxycarbonylphenoxy group were synthesized, separated, purified and identified. Then their structural pattern plots were also established by EI-MS and NMR data for the prediction of their skeleton types. There have been no reports on the synthesis and structural pattern recognition of compounds $\mathbf{1 F}-\mathbf{1 3 F}$; therefore, this study is an innovative application of a method. Finally, the synthesis and structural pattern recognition of compounds $\mathbf{1 F}-\mathbf{1 3 F}$ are necessary for drug discoveries.

\section{Experimental}

Solvents and Chemicals Deionized water was obtained from the Ultrapure Water System (Putity-UV, Suntex Instruments Corporation, Ltd., Taipei, Taiwan, R.O.C.). Methyl salicylate (1A), 5-nitrofurfural, cesium fluoride $(\mathrm{CsF})$ and $60 \%$ sodium hydride $(\mathrm{NaH})$ were purchased from Lancaster (Lancashire, U.K.). Potassium bromide (KBr) and 2-hydroxy-6-methoxybenzoic acid were purchased from Sigma-Aldrich (Shanghai, China). Methyl iodide $\left(\mathrm{CH}_{3} \mathrm{I}\right)$, sodium hydrogen carbonate $\left(\mathrm{NaHCO}_{3}\right), \mathrm{N}, \mathrm{N}$-dimethylformamide (DMF), dimethyl sulfoxide (DMSO), chloroform- $d_{1}\left(\mathrm{CDCl}_{3}\right)$ with tetramethylsilane (TMS) for NMR spectroscopy and $80 \% \mathrm{NaH}$ were purchased from Merck (Darmstadt, Germany). Ethyl acetate, $n$-hexane, chloroform and methanol were purchased from Mallinckrodt Baker (Phillipsburg, U.S.A.). Potassium hydroxide (KOH) and anhydrous magnesium sulfate were purchased from Shimakyu's Pure Chemical Corporation (Osaka, Japan). Benzene was purchased from Uni-Onward Corporation (Taipei, Taiwan, R.O.C.). The 2-hydroxy-6-methylbenzoic acid ethyl ester (5A) was purchased from TCI (Tokyo, Japan). All the starting materials except 2-hydroxy-6-methoxybenzoic acid methyl ester (9A), substituted salicylic acid 
methyl esters (2A-4A, 6A-8A and $\mathbf{1 0 A}-13 \mathrm{~A})$ (structures $\mathbf{1 A}-\mathbf{1 3 A}$ see Fig. 2) and ethyl 5-nitro-2-furoate, were synthesized as described in ref. 2.

Synthesis of 2-Hydroxy-6-methoxybenzoic Acid Methyl Ester (9A) A solution of $26.90 \mathrm{~g}(0.16 \mathrm{~mol})$ of 2-hydroxy-6-methoxybenzoic acid, $34.46 \mathrm{~g}(0.24 \mathrm{~mol})$ of $\mathrm{CsF}$ and $34.06 \mathrm{~g}(0.24 \mathrm{~mol})$ of $\mathrm{CH}_{3} \mathrm{I}$ in DMF $(250 \mathrm{ml})$ was mixed and stirred at room temperature for $24 \mathrm{~h}$. The reaction mixture was filtered. The filtrate was extracted with ethyl acetate and then washed with $5 \% \mathrm{NaHCO}_{3}$. The ethyl acetate layer was dried over anhydrous magnesium sulfate. The ethyl acetate was removed by rotary evaporation and the residue was then subjected to column chromatography with mobile phase consisting of a mixture of ethyl acetate- $n$-hexane $(1: 8, \mathrm{v} / \mathrm{v})$ to obtain pure compound 9A (synthetic scheme see Fig. 1). ${ }^{2,18)}$

Synthesis of Ethyl 5-(2'-Methoxycarbonyl-3'-methoxyphenoxy)furan2-carboxylate (9C) In comparison with compounds $\mathbf{1 F}-\mathbf{1 3 F}$, compound 9C was synthesized. To a vigorously stirred solution of $9.11 \mathrm{~g}(0.05 \mathrm{~mol})$ of 9A, DMSO $(50 \mathrm{ml})$ and $1.50 \mathrm{~g}(0.05 \mathrm{~mol})$ of $80 \% \mathrm{NaH}$, a solution of $7.59 \mathrm{~g}$ $(0.041 \mathrm{~mol})$ of ethyl 5-nitro-2-furoate and DMSO $(50 \mathrm{ml})$ was added dropwise. The reaction solution was stirred at $84-86^{\circ} \mathrm{C}$ for $24 \mathrm{~h}$ and the reaction mixture was then poured into ice water. The oily product was extracted with chloroform. The chloroform extract was washed with $5 \% \mathrm{KOH}$ and water, and was dried over anhydrous magnesium sulfate. The chloroform was concentrated by rotary evaporation and the residue was subjected to column chromatography with mobile phase consisting of a mixture of ethyl acetate- $n$-hexane $(1: 4, \mathrm{v} / \mathrm{v})$ to obtain pure compound $\left.9 \mathrm{C}^{2,19}\right)$

Synthesis of 5-(2'-Alkoxycarbonyl-substituted phenoxy)furfural Derivatives $(1 \mathrm{~F}-13 \mathrm{~F}) \quad$ A facile synthetic route is that 5-nitrofurfural reacted with corresponding salicylates. To a vigorously stirred solution of $0.12 \mathrm{~mol}$ of compounds 1A-13A, DMSO $(150 \mathrm{ml})$ and $4.8 \mathrm{~g}(0.12 \mathrm{~mol})$ of $60 \% \mathrm{NaH}$, a solution of $14.11 \mathrm{~g}(0.10 \mathrm{~mol})$ of 5-nitrofurfural and DMSO $(90 \mathrm{ml})$ was<smiles>COc1cccc(O)c1C(=O)O</smiles><smiles>COc1cccc(O)c1C(C)=O</smiles>

(9A)

Fig. 1. Synthetic Scheme of the 2-Hydroxy-6-methoxybenzoic Acid Methyl Ester (9A)<smiles>[R6]C(=O)c1c([R])c([R9])c([R9])c(O)c1[2H]</smiles><smiles>[R9]c1c([R9])c(Oc2ccc(C=O)o2)c(C(=O)O)c([R])c1[18OH]</smiles>

(1F-13F)

\begin{tabular}{cccccc}
\hline \hline Compound & $\mathrm{R}$ & $\mathrm{R}_{1}$ & $\mathrm{R}_{2}$ & $\mathrm{R}_{3}$ & $\mathrm{R}_{4}$ \\
\hline $\mathbf{1 A}(\mathbf{1 F})$ & $\mathrm{CH}_{3}$ & $\mathrm{H}$ & $\mathrm{H}$ & $\mathrm{H}$ & $\mathrm{H}$ \\
\hline $\mathbf{2 A}(\mathbf{2 F})$ & $\mathrm{CH}_{3}$ & $\mathrm{H}$ & $\mathrm{H}$ & $\mathrm{H}$ & $\mathrm{CH}_{3}$ \\
\hline $\mathbf{3 A}(\mathbf{3 F})$ & $\mathrm{CH}_{3}$ & $\mathrm{H}$ & $\mathrm{H}$ & $\mathrm{CH}_{3}$ & $\mathrm{H}$ \\
\hline $\mathbf{4 A}(\mathbf{4 F})$ & $\mathrm{CH}_{3}$ & $\mathrm{H}$ & $\mathrm{CH}_{3}$ & $\mathrm{H}$ & $\mathrm{H}$ \\
\hline $\mathbf{5 A}(\mathbf{5 F})$ & $\mathrm{C}_{2} \mathrm{H}_{5}$ & $\mathrm{CH}_{3}$ & $\mathrm{H}$ & $\mathrm{H}$ & $\mathrm{H}$ \\
\hline $\mathbf{6 A}(\mathbf{6 F})$ & $\mathrm{CH}_{3}$ & $\mathrm{H}$ & $\mathrm{H}$ & $\mathrm{H}$ & $\mathrm{OCH}_{3}$ \\
\hline $\mathbf{7 A}(\mathbf{7 F})$ & $\mathrm{CH}_{3}$ & $\mathrm{H}$ & $\mathrm{H}$ & $\mathrm{OCH}_{3}$ & $\mathrm{H}$ \\
\hline $\mathbf{8 A}(\mathbf{8 F})$ & $\mathrm{CH}_{3}$ & $\mathrm{H}$ & $\mathrm{OCH}_{3}$ & $\mathrm{H}$ & $\mathrm{H}$ \\
\hline $\mathbf{9 A}(\mathbf{9 F})$ & $\mathrm{CH}_{3}$ & $\mathrm{OCH}_{3}$ & $\mathrm{H}$ & $\mathrm{H}$ & $\mathrm{H}$ \\
\hline $\mathbf{1 0 A}(\mathbf{1 0 F})$ & $\mathrm{CH}_{3}$ & $\mathrm{H}$ & $\mathrm{H}$ & $\mathrm{Cl}$ & $\mathrm{H}$ \\
\hline $\mathbf{1 1} \mathbf{( 1 1 F})$ & $\mathrm{CH}_{3}$ & $\mathrm{H}$ & $\mathrm{Cl}$ & $\mathrm{H}$ & $\mathrm{H}$ \\
\hline $\mathbf{1 2 A}(\mathbf{1 2 F})$ & $\mathrm{CH}_{3}$ & $\mathrm{H}$ & $\mathrm{Br}$ & $\mathrm{H}$ & $\mathrm{H}$ \\
\hline $\mathbf{1 3 A}(\mathbf{1 3 F})$ & $\mathrm{CH}_{3}$ & $\mathrm{H}$ & $\mathrm{I}$ & $\mathrm{H}$ & $\mathrm{H}$ \\
\hline \hline
\end{tabular}

Fig. 2. Chemical Structures of the Substituted Salicylic Acid Methyl Esters $(\mathbf{1 A}-\mathbf{1 3 A})$ and Synthetic Scheme of the 5-(2'-Alkoxycarbonyl-substituted phenoxy)furfural Derivatives $(\mathbf{1 F}-\mathbf{1 3 F})$ added dropwise. The reaction solution was stirred at room temperature for $23 \mathrm{~h}$ and the reaction mixture was then poured into ice water. The oily product was extracted with chloroform. The chloroform extract was washed with $5 \% \mathrm{KOH}$ and water, and was dried over anhydrous magnesium sulfate. The chloroform was concentrated by rotary evaporation and the residue was subjected to column chromatography with different solvent systems (see Results) to obtain pure compounds $\mathbf{1 F}-\mathbf{1 3 F}$ (synthetic scheme see Fig. 2). ${ }^{20}$ )

Chromatography and Equipments The TLC experiment was performed on aluminium sheets (20 by $20 \mathrm{~cm}^{2}$; Art. 5554; Merck, Darmstadt, Germany) precoated with silica gel $60 \mathrm{~F}_{254}$. The TLC plates $\left(2\right.$ by $\left.5 \mathrm{~cm}^{2}\right)$ were developed in 10-ml solvent systems. The optimal solvent systems of the TLC plates were applied to the column chromatography method further. Column chromatography separations were achieved employing Silica Gel 60 (Kieselgel 60, 70-230 mesh; Merck, Darmstadt, Germany). A column was prepared by slurry packing the Silica Gel 60 sorbent in the optimal solvent system of the TLC plate. The residues were eluted using the optimal mobile phase composition of the TLC plate by a series of chromatography procedures. Each chromatographic experiment was completed until pure compounds $\mathbf{9 A}, \mathbf{9 C}$ and $\mathbf{1 F}-\mathbf{1 3 F}$ were obtained.

The melting point (mp) of the compounds were determined on a MP500D micro-melting point apparatus (Yanaco Company, Kyoto, Japan). The IR spectra were obtained in KBr discs on a Nicolet Impact-400 FT-IR spectrophotometer (Labequip Ltd., Ontario, Canada) in the $400-4000 \mathrm{~cm}^{-1}$ region. ${ }^{1} \mathrm{H}$ - and ${ }^{13} \mathrm{C}-\mathrm{NMR}$ spectra were obtained at $200 \mathrm{MHz}$ and $50 \mathrm{MHz}$ using a Bruker DPX-200 FT-NMR spectrometer (Bruker BioSpin GmbH, Rheinstetten, Germany) respectively. Chemical shifts are given on the $\delta \mathrm{ppm}$ scale downfield from TMS as an internal standard. The UV-vis spectra of the compounds (MeOH solution) were taken on a UV-visible-light recording spectrophotometer (UV-160A; Shimadzu Corporation, Kyoto, Japan). The EI-MS spectra were taken on a Hewlett-Packard benchtop model 5995 GC-MS (Hewlett-Packard, Palo Alto, CA, U.S.A.) under a $70 \mathrm{eV}$ ionisation potential. A HP-20, $25 \mathrm{~m} \times 0.32 \mathrm{~mm}$ (i.d.) capillary column was used for GC-MS. The column temperature was programmed from 110 to $300^{\circ} \mathrm{C}$ at a rate of $5^{\circ} \mathrm{C} / \mathrm{min}$. Helium was used as the carrier gas at a flow-rate of $1 \mathrm{ml} / \mathrm{min}$. Elemental analyses $(\mathrm{C}$ and $\mathrm{H})$ were performed on a Perkin-Elmer 2400 Series II CHNS/O Analyzer (Perkin-Elmer Corporation, Boston, MA, U.S.A.)

Identification and Pattern Recognition Analysis of 5-(2'-Alkoxycarbonyl-substituted phenoxy)furfural Derivatives $(\mathbf{1 F}-13 \mathrm{~F})$ Compounds 9A, 9C and 1F-13F were elucidated and identified by the spectral data (EIMS, UV, IR, ${ }^{1} \mathrm{H}$ - and $\left.{ }^{13} \mathrm{C}-\mathrm{NMR}\right)$. For comparison of the EI-MS and NMR patterns, an Excel worksheet was constructed with the codification of compounds $1 \mathrm{C}-\mathbf{1 3 C}$ (structure see Fig. 3) and $\mathbf{1 F}-\mathbf{1 3 F}$ with their respective EI-MS and NMR data. In the worksheet, fragmented and molecular ions, and chemical shifts of carbon and hydrogen were placed in their corresponding columns. The simple Excel worksheet analysis method can be suitable for any size of data set by a minimum of human input. Upon construction of the worksheet, the next step involves representing it on the EI-MS and NMR pattern plots.<smiles>[R]OC(=O)c1c([R])c([R])c([R])c([R])c1Oc1ccc(C(=O)OCC)o1</smiles>

(1C-13C)

\begin{tabular}{cccccc}
\hline \hline Compound & $\mathrm{R}$ & $\mathrm{R}_{1}$ & $\mathrm{R}_{2}$ & $\mathrm{R}_{3}$ & $\mathrm{R}_{4}$ \\
\hline $\mathbf{1 C}$ & $\mathrm{CH}_{3}$ & $\mathrm{H}$ & $\mathrm{H}$ & $\mathrm{H}$ & $\mathrm{H}$ \\
\hline $\mathbf{2 C}$ & $\mathrm{CH}_{3}$ & $\mathrm{H}$ & $\mathrm{H}$ & $\mathrm{H}$ & $\mathrm{CH}_{3}$ \\
\hline $\mathbf{3 C}$ & $\mathrm{CH}_{3}$ & $\mathrm{H}$ & $\mathrm{H}$ & $\mathrm{CH}_{3}$ & $\mathrm{H}$ \\
\hline $\mathbf{4 C}$ & $\mathrm{CH}_{3}$ & $\mathrm{H}$ & $\mathrm{CH}_{3}$ & $\mathrm{H}$ & $\mathrm{H}$ \\
\hline $\mathbf{5 C}$ & $\mathrm{C}_{2} \mathrm{H}_{5}$ & $\mathrm{CH}_{3}$ & $\mathrm{H}$ & $\mathrm{H}$ & $\mathrm{H}$ \\
\hline $\mathbf{6 C}$ & $\mathrm{CH}_{3}$ & $\mathrm{H}$ & $\mathrm{H}$ & $\mathrm{H}$ & $\mathrm{OCH}_{3}$ \\
\hline $\mathbf{7 C}$ & $\mathrm{CH}_{3}$ & $\mathrm{H}$ & $\mathrm{H}$ & $\mathrm{OCH}_{3}$ & $\mathrm{H}$ \\
\hline $\mathbf{8 C}$ & $\mathrm{CH}_{3}$ & $\mathrm{H}$ & $\mathrm{OCH}$ & $\mathrm{H}$ & $\mathrm{H}$ \\
\hline $\mathbf{9 C}$ & $\mathrm{CH}_{3}$ & $\mathrm{OCH}$ & $\mathrm{H}$ & $\mathrm{H}$ & $\mathrm{H}$ \\
\hline $\mathbf{1 0 C}$ & $\mathrm{CH}_{3}$ & $\mathrm{H}$ & $\mathrm{H}$ & $\mathrm{Cl}$ & $\mathrm{H}$ \\
\hline $\mathbf{1 1 C}$ & $\mathrm{CH}_{3}$ & $\mathrm{H}$ & $\mathrm{Cl}$ & $\mathrm{H}$ & $\mathrm{H}$ \\
\hline $\mathbf{1 2 C}$ & $\mathrm{CH}_{3}$ & $\mathrm{H}$ & $\mathrm{Br}$ & $\mathrm{H}$ & $\mathrm{H}$ \\
\hline $\mathbf{1 3 C}$ & $\mathrm{CH}_{3}$ & $\mathrm{H}$ & $\mathrm{I}$ & $\mathrm{H}$ & $\mathrm{H}$ \\
\hline \hline
\end{tabular}

Fig. 3. Chemical Structures of the Ethyl 5-(2'-Alkoxycarbonyl-substituted phenoxy)furan-2-carboxylates $(\mathbf{1 C}-\mathbf{1 3 C})$ 


\section{Results}

Analysis and Identification of 2-Hydroxy-6-methoxybenzoic Acid Methyl Ester (9A) Column chromatography with mobile phase consisting of a mixture of ethyl acetate$n$-hexane $(1: 8, \mathrm{v} / \mathrm{v})$; yield $24.50 \mathrm{~g}(84.1 \%)$. White solid; $\mathrm{mp} 46.5-47.5^{\circ} \mathrm{C}$. EI-MS $\mathrm{m} / \mathrm{z}$ (relative intensity \%): 182 $[\mathrm{M}]^{+}$(29.9), 107 (100). ${ }^{1} \mathrm{H}-\mathrm{NMR}\left(\mathrm{CDCl}_{3}\right) \delta: 3.84(3 \mathrm{H}, \mathrm{s}$, $\left.\mathrm{COOCH}_{3}\right), 3.93\left(3 \mathrm{H}, \mathrm{s}, \mathrm{OCH}_{3}-6\right), 6.39(1 \mathrm{H}, \mathrm{d}, J=8.3 \mathrm{~Hz}, \mathrm{H}-$ 3), 6.57 (1H, d, $J=8.3 \mathrm{~Hz}, \mathrm{H}-5), 7.31(1 \mathrm{H}, \mathrm{t}, J=8.3 \mathrm{~Hz}, \mathrm{H}-4)$, $11.48(1 \mathrm{H}, \mathrm{s}, \mathrm{OH}) .{ }^{13} \mathrm{C}-\mathrm{NMR}\left(\mathrm{CDCl}_{3}\right) \delta: 52.4\left(\mathrm{COOCH}_{3}\right)$, $56.1\left(\mathrm{OCH}_{3}-6\right), 102.2$ (C-1), 103.0 (C-5), 110.1 (C-3), 135.1 (C-4), 160.8 (C-2), 163.5 (C-6), 171.6 (CO). Anal. Calcd for $\mathrm{C}_{9} \mathrm{H}_{10} \mathrm{O}_{4}$ : C, 59.34; H, 5.53. Found: C, 59.26; H, 5.62.

Analysis and Identification of Ethyl 5-(2'-Methoxycarbonyl-3'-methoxyphenoxy)furan-2-carboxylate (9C) Column chromatography with mobile phase consisting of a mixture of ethyl acetate- $n$-hexane $(1: 4, \mathrm{v} / \mathrm{v})$; yield $4.10 \mathrm{~g}$ $(25.6 \%)$. Yellow liquid. EI-MS $\mathrm{m} / \mathrm{z}$ (relative intensity \%): $320\left[\mathrm{M}^{+}(67.2), 150(100) . \mathrm{UV} \lambda_{\max }(\mathrm{MeOH}) \mathrm{nm}(\log \varepsilon)\right.$ : 273 (4.36), 206 (4.34). IR $(\mathrm{KBr}) \mathrm{cm}^{-1}: 1721(\mathrm{C}=\mathrm{O}) .{ }^{1} \mathrm{H}-$ $\mathrm{NMR}\left(\mathrm{CDCl}_{3}\right) \delta: 1.32\left(3 \mathrm{H}, \mathrm{t}, J=7.1 \mathrm{~Hz}, \mathrm{COOCH}_{2} \mathrm{CH}_{3}\right), 3.83$ $\left(3 \mathrm{H}, \mathrm{s}, \mathrm{COOCH}_{3}\right), 3.85\left(3 \mathrm{H}, \mathrm{s}, \mathrm{OCH}_{3}-3^{\prime}\right), 4.30(2 \mathrm{H}, \mathrm{q}$, $\left.J=7.1 \mathrm{~Hz}, \mathrm{COOCH}_{2} \mathrm{CH}_{3}\right), 5.56(1 \mathrm{H}, \mathrm{d}, J=3.6 \mathrm{~Hz}, \mathrm{H}-4), 6.64$ $\left(1 \mathrm{H}, \mathrm{d}, J=8.4 \mathrm{~Hz}, \mathrm{H}-6^{\prime}\right), 6.74\left(1 \mathrm{H}, \mathrm{d}, J=8.4 \mathrm{~Hz}, \mathrm{H}-4^{\prime}\right), 7.10$ $(1 \mathrm{H}, \mathrm{d}, J=3.6 \mathrm{~Hz}, \mathrm{H}-3), 7.30\left(1 \mathrm{H}, \mathrm{t}, J=8.4 \mathrm{~Hz}, \mathrm{H}-5^{\prime}\right) .{ }^{13} \mathrm{C}-$ NMR $\left(\mathrm{CDCl}_{3}\right) \delta: 14.3\left(\mathrm{COOCH}_{2} \underline{\mathrm{CH}}_{3}\right), 52.6\left(\mathrm{COOCH}_{3}\right)$, $56.2\left(\mathrm{OCH}_{3}-3^{\prime}\right), 60.8\left(\mathrm{COOCH}_{2} \mathrm{CH}_{3}\right), 90.8(\mathrm{C}-4), 107.7(\mathrm{C}-$ $\left.4^{\prime}\right), 110.0\left(\mathrm{C}-6^{\prime}\right), 115.2\left(\mathrm{C}-2^{\prime}\right), 120.3(\mathrm{C}-3), 131.4\left(\mathrm{C}-5^{\prime}\right)$, $136.6(\mathrm{C}-2), 152.8\left(\mathrm{COOCH}_{2} \mathrm{CH}_{3}\right), 157.7\left(\mathrm{C}-1^{\prime}\right), 158.2(\mathrm{C}-$ $\left.3^{\prime}\right), 158.9(\mathrm{C}-5), 165.1 \quad\left(\mathrm{COOCH}_{3}\right)$. Anal. Calcd for $\mathrm{C}_{16} \mathrm{H}_{16} \mathrm{O}_{7}$ : C, 60.00; H, 5.04. Found: C, 60.06; H, 4.97.

Analysis and Identification of 5-(2'-Alkoxycarbonylsubstituted phenoxy)furfural Derivatives (1F-13F). 5(2'-Methoxycarbonylphenoxy)furfural (1F) Column chromatography with chloroform; yield $3.84 \mathrm{~g}$ (13.0\%). Brown liquid. EI-MS $m / z$ (relative intensity \%): $246[\mathrm{M}]^{+}$ (19.0), 126 (100). UV $\lambda_{\text {max }}(\mathrm{MeOH}) \mathrm{nm}(\log \varepsilon): 302$ (4.60), 209 (4.36). IR (KBr) cm ${ }^{-1}: 1729(\mathrm{C}=\mathrm{O}), 1675$ (CHO). ${ }^{1} \mathrm{H}-$ NMR $\left(\mathrm{CDCl}_{3}\right) \delta: 3.82\left(3 \mathrm{H}, \mathrm{s}, \mathrm{COOCH}{ }_{3}\right), 5.44(1 \mathrm{H}, \mathrm{d}$, $J=3.7 \mathrm{~Hz}, \mathrm{H}-4), 7.20(1 \mathrm{H}, \mathrm{d}, J=3.7 \mathrm{~Hz}, \mathrm{H}-3), 7.25(1 \mathrm{H}, \mathrm{d}$, $\left.J=1.1 \mathrm{~Hz}, \mathrm{H}-6^{\prime}\right), 7.35$ (1H, ddd, $\left.J=7.7,1.1 \mathrm{~Hz}, \mathrm{H}-4^{\prime}\right), 7.58$ $\left(1 \mathrm{H}, \mathrm{ddd}, J=7.7,1.8 \mathrm{~Hz}, \mathrm{H}-5^{\prime}\right), 7.99(1 \mathrm{H}, \mathrm{dd}, J=7.7,1.8 \mathrm{~Hz}$, $\left.\mathrm{H}-3^{\prime}\right), 9.37$ (1H, s, CHO). ${ }^{13} \mathrm{C}-\mathrm{NMR}\left(\mathrm{CDCl}_{3}\right) \quad \delta: 52.4$ $\left(\mathrm{COOCH} \underline{-1}_{3}\right), 89.0(\mathrm{C}-4), 121.6(\mathrm{C}-3), 123.0\left(\mathrm{C}-2^{\prime}\right), 125.3(\mathrm{C}-$ $\left.6^{\prime}\right), 126.3\left(\mathrm{C}-4^{\prime}\right), 132.3\left(\mathrm{C}-3^{\prime}\right), 134.1\left(\mathrm{C}-5^{\prime}\right), 144.8(\mathrm{C}-2)$, $152.7\left(\mathrm{C}-1^{\prime}\right), 163.1(\mathrm{C}-5), 164.8\left(\underline{C O O C H}_{3}\right), 175.6(\underline{\mathrm{CHO}})$. Anal. Calcd for $\mathrm{C}_{13} \mathrm{H}_{10} \mathrm{O}_{5}$ : C, 63.42; H, 4.09. Found: C, 63.35; H, 4.14.

5-(2'-Methoxycarbonyl-6' -methylphenoxy)furfural (2F) Column chromatography with chloroform; yield $3.71 \mathrm{~g}$ (11.9\%). Brown liquid. EI-MS $m / z$ (relative intensity \%): 260 $\left[\mathrm{M}^{+}\right.$(39.2), 134 (100). UV $\lambda_{\max }(\mathrm{MeOH}) \mathrm{nm}(\log \varepsilon): 302$ (4.58). IR (KBr) cm ${ }^{-1}: 1736(\mathrm{C}=\mathrm{O}), 1670(\mathrm{CHO}) .{ }^{1} \mathrm{H}-\mathrm{NMR}$ $\left(\mathrm{CDCl}_{3}\right) \delta: 2.27\left(3 \mathrm{H}, \mathrm{s}, \mathrm{CH}_{3}-6^{\prime}\right), 3.77\left(3 \mathrm{H}, \mathrm{s}, \mathrm{COOCH} \underline{\mathrm{H}}_{3}\right)$, $5.23(1 \mathrm{H}, \mathrm{d}, J=3.8 \mathrm{~Hz}, \mathrm{H}-4), 7.18(1 \mathrm{H}, \mathrm{d}, J=3.8 \mathrm{~Hz}, \mathrm{H}-3)$, $7.29\left(1 \mathrm{H}, \mathrm{t}, J=7.6 \mathrm{~Hz}, \mathrm{H}-4^{\prime}\right), 7.48(1 \mathrm{H}, \mathrm{dd}, J=7.6,1.7 \mathrm{~Hz}, \mathrm{H}-$ $\left.5^{\prime}\right), 7.83\left(1 \mathrm{H}, \mathrm{dd}, J=7.6,1.7 \mathrm{~Hz}, \mathrm{H}-3^{\prime}\right), 9.34(1 \mathrm{H}, \mathrm{s}, \mathrm{CHO})$. ${ }^{13} \mathrm{C}-\mathrm{NMR}\left(\mathrm{CDCl}_{3}\right) \delta: 15.9\left(\mathrm{CH}_{3}-6^{\prime}\right), 52.4\left(\mathrm{COOCH}_{3}\right), 87.0$ (C-4), 123.7 (C-3), 126.5 (C-4'), 126.5 (C-6'), 130.0 (C-3'), 132.1 (C-2'), $136.0\left({\mathrm{C}-5^{\prime}}^{\prime}\right), 144.5$ (C-2), 150.3 (C-1'), 163.6

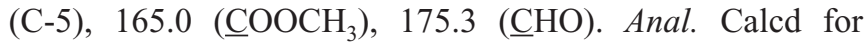

$\mathrm{C}_{14} \mathrm{H}_{12} \mathrm{O}_{5}$ : C, 64.61; H, 4.65. Found: C, 64.55; H, 4.73.

5-(2'-Methoxycarbonyl-5'-methylphenoxy)furfural (3F) Column chromatography with chloroform; yield $3.16 \mathrm{~g}$ (10.1\%). Brown solid; mp 60.5-61.5 ${ }^{\circ} \mathrm{C}$. EI-MS $\mathrm{m} / \mathrm{z}$ (relative intensity \%): $260[\mathrm{M}]^{+}(41.0), 134$ (100). UV $\lambda_{\max }$ $(\mathrm{MeOH}) \mathrm{nm}(\log \varepsilon): 301$ (4.79), 231 (4.55), 208 (4.55). IR $(\mathrm{KBr}) \mathrm{cm}^{-1}: 1723(\mathrm{C}=\mathrm{O}), 1670(\mathrm{CHO}) .{ }^{1} \mathrm{H}-\mathrm{NMR}\left(\mathrm{CDCl}_{3}\right)$ $\delta: 2.38\left(3 \mathrm{H}, \mathrm{s}, \mathrm{CH}_{3}-5^{\prime}\right), 3.77\left(3 \mathrm{H}, \mathrm{s}, \mathrm{COOCH} \underline{(H}_{3}\right), 5.40(1 \mathrm{H}, \mathrm{d}$, $J=3.8 \mathrm{~Hz}, \mathrm{H}-4), 7.00$ (1H, s, H-6'), $7.13(1 \mathrm{H}, \mathrm{d}, J=8.0 \mathrm{~Hz}$, H-4' $), 7.18(1 \mathrm{H}, \mathrm{d}, J=3.8 \mathrm{~Hz}, \mathrm{H}-3), 7.87(1 \mathrm{H}, \mathrm{d}, J=8.0 \mathrm{~Hz}$, $\left.\mathrm{H}-3^{\prime}\right), 9.34(1 \mathrm{H}, \mathrm{s}, \mathrm{CHO}) .{ }^{13} \mathrm{C}-\mathrm{NMR}\left(\mathrm{CDCl}_{3}\right) \delta: 21.4\left(\mathrm{CH}_{3}-\right.$ $\left.5^{\prime}\right), 52.2\left(\mathrm{COOCH}_{3}\right), 88.9(\mathrm{C}-4), 119.9\left(\mathrm{C}-2^{\prime}\right), 122.2\left(\mathrm{C}-6^{\prime}\right)$, 125.4 (C-3), $127.2\left(\mathrm{C}-4^{\prime}\right), 132.3\left(\mathrm{C}-3^{\prime}\right), 144.7$ (C-2), 145.6 $\left(\mathrm{C}-5^{\prime}\right), 152.7\left(\mathrm{C}-1^{\prime}\right), 163.3(\mathrm{C}-5), 164.8\left(\mathrm{COOCH}_{3}\right), 175.5$ (ㅌO). Anal. Calcd for $\mathrm{C}_{14} \mathrm{H}_{12} \mathrm{O}_{5}$ : C, 64.61; H, 4.65. Found: C, 64.68; H, 4.70.

5-(2'-Methoxycarbonyl-4'-methylphenoxy)furfural (4F) Column chromatography with chloroform; yield $4.36 \mathrm{~g}$ $(14.0 \%)$. Brown liquid. EI-MS $m / z$ (relative intensity \%): 260 $\left[\mathrm{M}^{+}\right.$(27.5), 134 (100). UV $\lambda_{\max }(\mathrm{MeOH}) \mathrm{nm}(\log \varepsilon): 311$ (3.99), 238 (4.16), 211 (4.60). IR (KBr) cm $\mathrm{cm}^{-1}: 1729(\mathrm{C}=\mathrm{O})$, $1677(\mathrm{CHO}) .{ }^{1} \mathrm{H}-\mathrm{NMR}\left(\mathrm{CDCl}_{3}\right) \delta: 2.37\left(3 \mathrm{H}, \mathrm{s}, \mathrm{CH}_{3}-4^{\prime}\right), 3.78$ $(3 \mathrm{H}, \mathrm{s}, \mathrm{COOCH}-3), 5.36(1 \mathrm{H}, \mathrm{d}, J=3.7 \mathrm{~Hz}, \mathrm{H}-4), 7.10(1 \mathrm{H}, \mathrm{d}$, $\left.J=8.3 \mathrm{~Hz}, \mathrm{H}-6^{\prime}\right), 7.16(1 \mathrm{H}, \mathrm{d}, J=3.7 \mathrm{~Hz}, \mathrm{H}-3), 7.35(1 \mathrm{H}, \mathrm{dd}$, $\left.J=8.3,2.3 \mathrm{~Hz}, \mathrm{H}-5^{\prime}\right), 7.76\left(1 \mathrm{H}, \mathrm{d}, J=2.3 \mathrm{~Hz}, \mathrm{H}-3^{\prime}\right), 9.33$ $(1 \mathrm{H}, \mathrm{s}, \mathrm{CHO}) .{ }^{13} \mathrm{C}-\mathrm{NMR}\left(\mathrm{CDCl}_{3}\right) \delta: 20.6\left(\mathrm{CH}_{3}-4^{\prime}\right), 52.3$ $\left(\mathrm{COOCH} \underline{-1}_{3}\right), 88.5(\mathrm{C}-4), 121.6\left(\mathrm{C}-6^{\prime}\right), 122.6\left(\mathrm{C}-2^{\prime}\right), 125.4(\mathrm{C}-$

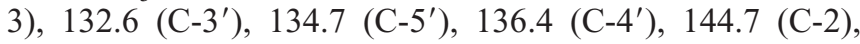
$150.4\left(\mathrm{C}-1^{\prime}\right), 163.6(\mathrm{C}-5), 165.0\left(\mathrm{COOCH}_{3}\right), 175.5(\underline{\mathrm{CHO}})$. Anal. Calcd for $\mathrm{C}_{14} \mathrm{H}_{12} \mathrm{O}_{5}: \mathrm{C}, 64.61 ; \mathrm{H}, 4.65$. Found: $\mathrm{C}$, 64.69; H, 4.59.

5-(2'-Ethoxycarbonyl-3'-methylphenoxy)furfural (5F) Column chromatography with chloroform; yield $6.23 \mathrm{~g}$ (18.9\%). Yellow liquid. EI-MS $\mathrm{m} / \mathrm{z}$ (relative intensity \%): $274[\mathrm{M}]^{+}$(30.6), $112(100) . \mathrm{UV} \lambda_{\max }(\mathrm{MeOH}) \mathrm{nm}(\log \varepsilon)$ : 301 (4.39), 210 (4.58). IR (KBr) cm $\mathrm{cm}^{-1}: 1736(\mathrm{C}=\mathrm{O}), 1677$ (CHO). ${ }^{1} \mathrm{H}-\mathrm{NMR} \quad\left(\mathrm{CDCl}_{3}\right) \quad \delta: 1.23 \quad(3 \mathrm{H}, \quad \mathrm{t}, \quad J=7.1 \mathrm{~Hz}$, $\left.\mathrm{COOCH}_{2} \mathrm{CH}_{3}\right), 2.36\left(3 \mathrm{H}, \mathrm{s}, \mathrm{CH}_{3}-3^{\prime}\right), 4.29(2 \mathrm{H}, \mathrm{q}, J=7.1 \mathrm{~Hz}$, $\left.\mathrm{COOCH}_{2} \mathrm{CH}_{3}\right), 5.48(1 \mathrm{H}, \mathrm{d}, J=3.7 \mathrm{~Hz}, \mathrm{H}-4), 6.99(1 \mathrm{H}, \mathrm{d}$, $\left.J=7.9 \mathrm{~Hz}, \mathrm{H}-6^{\prime}\right), 7.10\left(1 \mathrm{H}, \mathrm{d}, J=7.9 \mathrm{~Hz}, \mathrm{H}-4^{\prime}\right), 7.17(1 \mathrm{H}, \mathrm{d}$, $J=3.7 \mathrm{~Hz}, \mathrm{H}-3), 7.30\left(1 \mathrm{H}, \mathrm{t}, J=7.9 \mathrm{~Hz}, \mathrm{H}-5^{\prime}\right), 9.35(1 \mathrm{H}, \mathrm{s}$, CHO). ${ }^{13} \mathrm{C}-\mathrm{NMR}\left(\mathrm{CDCl}_{3}\right) \quad \delta: 14.0\left(\mathrm{COOCH}_{2} \underline{\mathrm{CH}}_{3}\right), 19.5$ $\left(\mathrm{CH}_{3}-3^{\prime}\right), 61.5\left(\mathrm{COOCH}{ }_{2} \mathrm{CH}_{3}\right), 89.6(\mathrm{C}-4), 117.1\left(\mathrm{C}-6^{\prime}\right)$, 125.0 (C-3), 126.2 (C-2'), $128.0\left(\mathrm{C}-4^{\prime}\right), 130.8$ (C-5'), 138.4 $\left(\mathrm{C}-3^{\prime}\right), 144.8$ (C-2), 150.9 (C-1'), 162.8 (C-5), 166.0 $\left(\underline{\mathrm{COOCH}} \mathrm{CH}_{3}\right), 175.6$ (ㄷO). Anal. Calcd for $\mathrm{C}_{15} \mathrm{H}_{14} \mathrm{O}_{5}: \mathrm{C}$, 65.69; H, 5.15. Found: C, 65.73; H, 5.09.

5-(2'-Methoxycarbonyl-6'-methoxyphenoxy)furfural (6F) Column chromatography with mobile phase consisting of a mixture of ethyl acetate- $n$-hexane $(1: 2, \mathrm{v} / \mathrm{v})$; yield $1.03 \mathrm{~g}(3.1 \%)$. Green solid; $\mathrm{mp} 79-80^{\circ} \mathrm{C}$. EI-MS $\mathrm{m} / z$ (relative intensity \%): $276[\mathrm{M}]^{+}(14.2), 150$ (100). UV $\lambda_{\max }$ $(\mathrm{MeOH}) \mathrm{nm}(\log \varepsilon): 305$ (4.65), 209 (4.60). IR $(\mathrm{KBr}) \mathrm{cm}^{-1}$ : $1736(\mathrm{C}=\mathrm{O}), 1670(\mathrm{CHO}) .{ }^{1} \mathrm{H}-\mathrm{NMR}\left(\mathrm{CDCl}_{3}\right) \delta: 3.81(3 \mathrm{H}, \mathrm{s}$, $\left.\mathrm{COOCH}_{3}\right), 3.83\left(3 \mathrm{H}, \mathrm{s}, \mathrm{OCH}_{3}-6^{\prime}\right), 5.35(1 \mathrm{H}, \mathrm{d}, J=3.8 \mathrm{~Hz}, \mathrm{H}-$ 4), $7.19(1 \mathrm{H}, \mathrm{d}, J=3.8 \mathrm{~Hz}, \mathrm{H}-3), 7.20(1 \mathrm{H}, \mathrm{dd}, J=8.0,1.7 \mathrm{~Hz}$, H-5'), $7.32\left(1 \mathrm{H}, \mathrm{t}, J=8.0 \mathrm{~Hz}, \mathrm{H}-4^{\prime}\right), 7.53(1 \mathrm{H}, \mathrm{dd}, J=8.0$, $\left.1.7 \mathrm{~Hz}, \mathrm{H}-3^{\prime}\right), 9.33(1 \mathrm{H}, \mathrm{s}, \mathrm{CHO}) .{ }^{13} \mathrm{C}-\mathrm{NMR}\left(\mathrm{CDCl}_{3}\right) \delta: 52.5$ $\left(\mathrm{COOCH}+\underline{\mathrm{C}}_{3}\right), 56.4\left(\mathrm{OCH}_{3}-6^{\prime}\right), 87.3(\mathrm{C}-4), 116.9\left(\mathrm{C}-5^{\prime}\right), 122.9$ $\left(\mathrm{C}-3^{\prime}\right), 125.0\left(\mathrm{C}-2^{\prime}\right), 127.0(\mathrm{C}-3), 127.0\left(\mathrm{C}-4^{\prime}\right), 141.1\left(\mathrm{C}-1^{\prime}\right)$, 144.3 (C-2), 152.1 (C-6'), 163.7 (C-5), $164.8\left(\right.$ COOCH$\left._{3}\right)$, 
175.3 (ㅌHO). Anal. Calcd for $\mathrm{C}_{14} \mathrm{H}_{12} \mathrm{O}_{6}:$ C, 60.87; H, 4.38. Found: C, 60.79; H, 4.34.

5-(2'-Methoxycarbonyl-5'-methoxyphenoxy)furfural (7F) Column chromatography with chloroform; yield $1.37 \mathrm{~g}(4.1 \%)$. Green solid; mp 99-100 ${ }^{\circ} \mathrm{C}$. EI-MS $m / z$ (relative intensity \%): $276[\mathrm{M}]^{+}(41.6), 63$ (100). UV $\lambda_{\max }$ $(\mathrm{MeOH}) \mathrm{nm}(\log \varepsilon): 301$ (4.64), 254 (4.50), 209 (4.51). IR $(\mathrm{KBr}) \mathrm{cm}^{-1}: 1723(\mathrm{C}=\mathrm{O}), 1670(\mathrm{CHO}) .{ }^{1} \mathrm{H}-\mathrm{NMR}\left(\mathrm{CDCl}_{3}\right)$ $\delta: 3.75\left(3 \mathrm{H}, \mathrm{s}, \mathrm{COOCH} \underline{\mathrm{H}}_{3}\right), 3.83\left(3 \mathrm{H}, \mathrm{s}, \mathrm{OCH}_{3}-5^{\prime}\right), 5.42(1 \mathrm{H}$, d, $J=3.7 \mathrm{~Hz}, \mathrm{H}-4), 6.70\left(1 \mathrm{H}, \mathrm{d}, J=2.5 \mathrm{~Hz}, \mathrm{H}-6^{\prime}\right), 6.83(1 \mathrm{H}$, $\left.\mathrm{dd}, J=8.8,2.5 \mathrm{~Hz}, \mathrm{H}-4^{\prime}\right), 7.18(1 \mathrm{H}, \mathrm{d}, J=3.7 \mathrm{~Hz}, \mathrm{H}-3), 7.97$ $\left(1 \mathrm{H}, \mathrm{d}, J=8.8 \mathrm{~Hz}, \mathrm{H}-3^{\prime}\right), 9.34$ (1H, s, CHO). ${ }^{13} \mathrm{C}-\mathrm{NMR}$ $\left(\mathrm{CDCl}_{3}\right) \delta: 52.1\left(\mathrm{COOCH}_{3}\right), 55.8\left(\mathrm{OCH}_{3}-5^{\prime}\right), 88.9(\mathrm{C}-4)$, $107.5\left(\mathrm{C}-6^{\prime}\right), 111.8\left(\mathrm{C}-4^{\prime}\right), 114.9\left(\mathrm{C}-2^{\prime}\right), 125.2$ (C-3), 134.0 (C-3'), 144.7 (C-2), $154.3\left(\mathrm{C}-1^{\prime}\right), 163.1$ (C-5'), 164.2 (C-5),

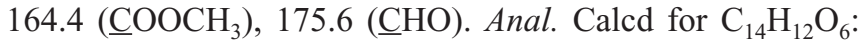
C, 60.87; H, 4.38. Found: C, 60.96; H, 4.33.

5-(2'-Methoxycarbonyl-4'-methoxyphenoxy)furfural (8F) Column chromatography with chloroform; yield $6.48 \mathrm{~g}(19.5 \%)$. Brown solid; $\mathrm{mp} 50.5-51.5^{\circ} \mathrm{C}$. EI-MS $m / z$

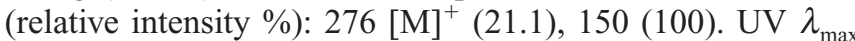
$(\mathrm{MeOH}) \mathrm{nm}(\log \varepsilon): 306$ (4.16), 209 (4.18). IR (KBr) $\mathrm{cm}^{-1}$ : $1729(\mathrm{C}=\mathrm{O}), 1664(\mathrm{CHO}) .{ }^{1} \mathrm{H}-\mathrm{NMR}\left(\mathrm{CDCl}_{3}\right) \delta: 3.78(3 \mathrm{H}, \mathrm{s}$, $\left.\mathrm{COOCH}_{3}\right), 3.83\left(3 \mathrm{H}, \mathrm{s}, \mathrm{OCH}_{3}-4^{\prime}\right), 5.31(1 \mathrm{H}, \mathrm{d}, J=3.8 \mathrm{~Hz}, \mathrm{H}-$ 4), $7.07\left(1 \mathrm{H}, \mathrm{dd}, J=9.0,3.0 \mathrm{~Hz}, \mathrm{H}-5^{\prime}\right), 7.14-7.18(2 \mathrm{H}, \mathrm{m}$, H-3, 6' $), 7.45$ (1H, d, J=3.0 Hz, H-3'), 9.32 (1H, s, CHO). ${ }^{13} \mathrm{C}-\mathrm{NMR}\left(\mathrm{CDCl}_{3}\right) \delta: 52.5\left(\mathrm{COOC \textrm {CH } _ { 3 }}\right), 55.9\left(\mathrm{OCH}_{3}-4^{\prime}\right), 88.0$ (C-4), 116.1 (C-3'), 120.1 (C-5'), 123.3 (C-6'), 123.7 (C-2'), 125.8 (C-3), 144.5 (C-2), 146.0 (C-1' $), 157.4$ (C-4'), 164.1 $(\mathrm{C}-5), 164.7\left(\mathrm{COOCH}_{3}\right), 175.4$ ( $\left.\underline{\mathrm{CHO}}\right)$. Anal. Calcd for $\mathrm{C}_{14} \mathrm{H}_{12} \mathrm{O}_{6}: \mathrm{C}, 60.87$; H, 4.38. Found: C, 60.80; H, 4.29.

5-(2'-Methoxycarbonyl-3'-methoxyphenoxy)furfural (9F) Column chromatography with mobile phase consisting of a mixture of ethyl acetate- $n$-hexane $(1: 2, \mathrm{v} / \mathrm{v})$; yield $7.82 \mathrm{~g}(23.6 \%)$. Yellow liquid. EI-MS $\mathrm{m} / \mathrm{z}$ (relative intensity $\%): 276[\mathrm{M}]^{+}(6.7), 55$ (100). UV $\lambda_{\text {max }}(\mathrm{MeOH}) \mathrm{nm}(\log \varepsilon)$ : 298 (4.79), 209 (4.61). IR (KBr) cm $\mathrm{cm}^{-1}: 1737(\mathrm{C}=\mathrm{O}), 1675$ (CHO). ${ }^{1} \mathrm{H}-\mathrm{NMR}\left(\mathrm{CDCl}_{3}\right) \delta: 3.83\left(3 \mathrm{H}, \mathrm{s}, \mathrm{COOC \underline {CH } _ { 3 }}\right), 3.84$ $\left(3 \mathrm{H}, \mathrm{s}, \mathrm{OCH}_{3}-3^{\prime}\right), 5.59(1 \mathrm{H}, \mathrm{d}, J=3.8 \mathrm{~Hz}, \mathrm{H}-4), 6.73(1 \mathrm{H}, \mathrm{d}$, $\left.J=8.4 \mathrm{~Hz}, \mathrm{H}-6^{\prime}\right), 6.80\left(1 \mathrm{H}, \mathrm{d}, J=8.4 \mathrm{~Hz}, \mathrm{H}-4^{\prime}\right), 7.18(1 \mathrm{H}, \mathrm{d}$, $J=3.8 \mathrm{~Hz}, \mathrm{H}-3), 7.35\left(1 \mathrm{H}, \mathrm{t}, J=8.4 \mathrm{~Hz}, \mathrm{H}-5^{\prime}\right), 9.37(1 \mathrm{H}, \mathrm{s}$, CHO). ${ }^{13} \mathrm{C}-\mathrm{NMR}\left(\mathrm{CDCl}_{3}\right) \delta: 52.7\left(\mathrm{COOCH}_{3}\right), 56.3\left(\mathrm{OCH}_{3}-\right.$

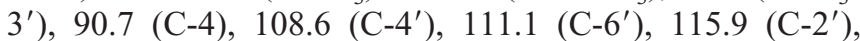
124.8 (C-3), 131.7 (C-5'), 145.0 (C-2), 151.8 (C-1'), 157.9 (C-3'), 161.9 (C-5), 164.8 ( COOCH$\left._{3}\right), 175.9$ (ㅌO). Anal. Calcd for $\mathrm{C}_{14} \mathrm{H}_{12} \mathrm{O}_{6}: \mathrm{C}, 60.87 ; \mathrm{H}, 4.38$. Found: $\mathrm{C}, 60.90 ; \mathrm{H}$, 4.43 .

5-(2'-Methoxycarbonyl-5'-chlorophenoxy)furfural (10F) Column chromatography with mobile phase consisting of a mixture of chloroform-benzene $(1: 1, \mathrm{v} / \mathrm{v})$; yield $1.07 \mathrm{~g}(3.2 \%)$. Green solid; $\mathrm{mp} 79.5-80.5^{\circ} \mathrm{C}$. EI-MS $\mathrm{m} / \mathrm{z}$ (relative intensity \%): $282[\mathrm{M}]^{+}+2(3.5), 280[\mathrm{M}]^{+}(10.5)$, 126 (100). UV $\lambda_{\max }(\mathrm{MeOH}) \mathrm{nm}(\log \varepsilon): 302$ (4.53), 235 (4.37), 206 (4.44). IR (KBr) cm $\mathrm{cm}^{-1}: 1721(\mathrm{C}=\mathrm{O}), 1667$ $(\mathrm{CHO}) .{ }^{1} \mathrm{H}-\mathrm{NMR}\left(\mathrm{CDCl}_{3}\right) \delta: 3.80\left(3 \mathrm{H}, \mathrm{s}, \mathrm{COOCH}{ }_{3}\right), 5.54$ $(1 \mathrm{H}, \mathrm{d}, J=3.8 \mathrm{~Hz}, \mathrm{H}-4), 7.19\left(1 \mathrm{H}, \mathrm{s}, \mathrm{H}-6^{\prime}\right), 7.21(1 \mathrm{H}, \mathrm{d}$, $J=3.8 \mathrm{~Hz}, \mathrm{H}-3), 7.31$ (1H, dd, $\left.J=8.5,2.0 \mathrm{~Hz}, \mathrm{H}-4^{\prime}\right), 7.93$ $\left(1 \mathrm{H}, \mathrm{d}, J=8.5 \mathrm{~Hz}, \mathrm{H}-3^{\prime}\right), 9.38(1 \mathrm{H}, \mathrm{s}, \mathrm{CHO}) .{ }^{13} \mathrm{C}-\mathrm{NMR}$ $\left(\mathrm{CDCl}_{3}\right) \delta: 52.6\left(\mathrm{COOCH}_{3}\right), 89.9(\mathrm{C}-4), 121.3\left(\mathrm{C}-2^{\prime}\right), 121.8$ (C-6'), $125.0(\mathrm{C}-3), 126.6\left(\mathrm{C}-4^{\prime}\right), 133.4\left(\mathrm{C}-3^{\prime}\right), 139.8\left(\mathrm{C}-5^{\prime}\right)$, 145.1 (C-2), $153.3\left(\mathrm{C}-1^{\prime}\right), 161.9(\mathrm{C}-5), 164.0\left(\mathrm{COOCH}_{3}\right)$,
175.7 (ㄷH). Anal. Calcd for $\mathrm{C}_{13} \mathrm{H}_{9} \mathrm{ClO}_{5}$ : C, 55.63; H, 3.23. Found: C, 55.71; H, 3.28.

5-(2'-Methoxycarbonyl-4' -chlorophenoxy)furfural (11F) Column chromatography with chloroform; yield $1.88 \mathrm{~g}(5.6 \%)$. Brown liquid. EI-MS $\mathrm{m} / \mathrm{z}$ (relative intensity \%): $282[\mathrm{M}]^{+}+2(2.9), 280[\mathrm{M}]^{+}(8.7), 126(100)$. UV $\lambda_{\max }$ $(\mathrm{MeOH}) \mathrm{nm}(\log \varepsilon): 301$ (4.71), 208 (4.61). IR $(\mathrm{KBr}) \mathrm{cm}^{-1}$ : $1729(\mathrm{C}=\mathrm{O}), 1675(\mathrm{CHO}) .{ }^{1} \mathrm{H}-\mathrm{NMR}\left(\mathrm{CDCl}_{3}\right) \delta: 3.81(3 \mathrm{H}, \mathrm{s}$, $\left.\mathrm{COOCH}_{3}\right), 5.48(1 \mathrm{H}, \mathrm{d}, J=3.7 \mathrm{~Hz}, \mathrm{H}-4), 7.15-7.20(2 \mathrm{H}, \mathrm{m}$, H-3, 6' $), 7.52\left(1 \mathrm{H}, \mathrm{dd}, J=8.7,2.7 \mathrm{~Hz}, \mathrm{H}-5^{\prime}\right), 7.95(1 \mathrm{H}, \mathrm{d}$, $\left.J=2.7 \mathrm{~Hz}, \mathrm{H}-3^{\prime}\right), 9.36$ (1H, s, CHO). ${ }^{13} \mathrm{C}-\mathrm{NMR}\left(\mathrm{CDCl}_{3}\right) \delta$ : $52.7\left(\mathrm{COOCH}_{3}\right), 89.3(\mathrm{C}-4), 123.0\left(\mathrm{C}-6^{\prime}\right), 124.3\left(\mathrm{C}-2^{\prime}\right)$, 125.2 (C-3), $131.8\left(\mathrm{C}-4^{\prime}\right), 132.1\left(\mathrm{C}-3^{\prime}\right), 134.0\left(\mathrm{C}-5^{\prime}\right), 144.9$ (C-2), $151.2\left(\mathrm{C}-1^{\prime}\right), 162.4(\mathrm{C}-5), 163.6\left(\mathrm{COOCH}_{3}\right), 175.6$ (CHO). Anal. Calcd for $\mathrm{C}_{13} \mathrm{H}_{9} \mathrm{ClO}_{5}: \mathrm{C}, 55.63 ; \mathrm{H}, 3.23$. Found: C, 55.59; H, 3.31.

5-(2'-Methoxycarbonyl-4'-bromophenoxy)furfural (12F) Column chromatography with mobile phase consisting of a mixture of chloroform-benzene $(1: 1, \mathrm{v} / \mathrm{v})$; yield $4.72 \mathrm{~g}(12.1 \%)$. Yellow liquid. EI-MS $\mathrm{m} / \mathrm{z}$ (relative intensity \%): $326[\mathrm{M}]^{+}+2(10.5), 324[\mathrm{M}]^{+}$(10.5), 126 (100). UV $\lambda_{\text {max }}(\mathrm{MeOH}) \mathrm{nm}(\log \varepsilon): 300$ (4.89), 212 (4.78). IR (KBr) $\mathrm{cm}^{-1}: 1737(\mathrm{C}=\mathrm{O}), 1667(\mathrm{CHO}) .{ }^{1} \mathrm{H}-\mathrm{NMR}\left(\mathrm{CDCl}_{3}\right) \delta: 3.81$ $(3 \mathrm{H}, \mathrm{s}, \mathrm{COOCH}-3), 5.48(1 \mathrm{H}, \mathrm{d}, J=3.7 \mathrm{~Hz}, \mathrm{H}-4), 7.10(1 \mathrm{H}, \mathrm{d}$, $\left.J=8.7 \mathrm{~Hz}, \mathrm{H}-6^{\prime}\right), 7.19(1 \mathrm{H}, \mathrm{d}, J=3.7 \mathrm{~Hz}, \mathrm{H}-3), 7.66(1 \mathrm{H}, \mathrm{dd}$, $\left.J=8.7,2.6 \mathrm{~Hz}, \mathrm{H}-5^{\prime}\right), 8.09\left(1 \mathrm{H}, \mathrm{d}, J=2.6 \mathrm{~Hz}, \mathrm{H}-3^{\prime}\right), 9.36$ $(1 \mathrm{H}, \mathrm{s}, \mathrm{CHO}) .{ }^{13} \mathrm{C}-\mathrm{NMR}\left(\mathrm{CDCl}_{3}\right) \delta: 52.7\left(\mathrm{COOCH}{ }_{3}\right), 89.4$ (C-4), $119.2\left(\mathrm{C}-4^{\prime}\right), 123.2\left(\mathrm{C}-6^{\prime}\right), 124.5\left(\mathrm{C}-2^{\prime}\right), 125.2(\mathrm{C}-3)$, 135.1 (C-3'), 137.0 (C-5'), 145.0 (C-2), 151.8 (C-1'), 162.3 (C-5), $163.5\left(\mathrm{COOCH}_{3}\right), 175.6$ ( $\left.\underline{\mathrm{CHO}}\right)$. Anal. Calcd for $\mathrm{C}_{13} \mathrm{H}_{9} \mathrm{BrO}_{5}$ : C, 48.03; H, 2.79. Found: C, 48.09; H, 2.85.

5-(2'-Methoxycarbonyl-4'-iodophenoxy)furfural (13F) Column chromatography with mobile phase consisting of a mixture of chloroform-benzene $(1: 1, \mathrm{v} / \mathrm{v})$; yield $5.45 \mathrm{~g}$ (12.2\%). Brown solid; mp 59.5-60.5 ${ }^{\circ} \mathrm{C}$. EI-MS $\mathrm{m} / \mathrm{z}$ (relative intensity \%): $372[\mathrm{M}]^{+}(15.3), 126$ (100). UV $\lambda_{\max }$ $(\mathrm{MeOH}) \mathrm{nm}(\log \varepsilon): 305$ (4.44), 218 (4.41). IR $(\mathrm{KBr}) \mathrm{cm}^{-1}$ : $1729(\mathrm{C}=\mathrm{O}), 1659(\mathrm{CHO}) .{ }^{1} \mathrm{H}-\mathrm{NMR}\left(\mathrm{CDCl}_{3}\right) \delta: 3.81(3 \mathrm{H}, \mathrm{s}$, $\left.\mathrm{COOCH}_{3}\right), 5.52(1 \mathrm{H}, \mathrm{d}, J=3.7 \mathrm{~Hz}, \mathrm{H}-4), 6.96(1 \mathrm{H}, \mathrm{d}$, $\left.J=8.6 \mathrm{~Hz}, \mathrm{H}-6^{\prime}\right), 7.19(1 \mathrm{H}, \mathrm{d}, J=3.7 \mathrm{~Hz}, \mathrm{H}-3), 7.84(1 \mathrm{H}, \mathrm{dd}$, $\left.J=8.6,2.3 \mathrm{~Hz}, \mathrm{H}-5^{\prime}\right), 8.27\left(1 \mathrm{H}, \mathrm{d}, J=2.3 \mathrm{~Hz}, \mathrm{H}-3^{\prime}\right), 9.38$

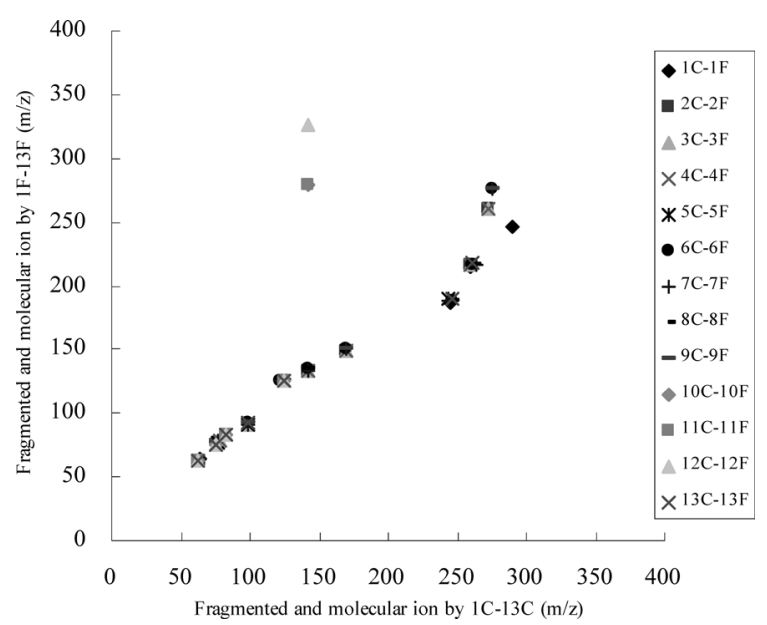

Fig. 4. Plot of Compounds $\mathbf{1 C}-\mathbf{1 3 C}$ and $\mathbf{1 F}-\mathbf{1 3 F}$ for Fragmented and Molecular Ions Generated as an Excel Worksheet Method

Each point represents fragmented and molecular ions of $\mathbf{1 C}-\mathbf{1 3 C}$ versus $\mathbf{1 F}-\mathbf{1 3 F}$. 


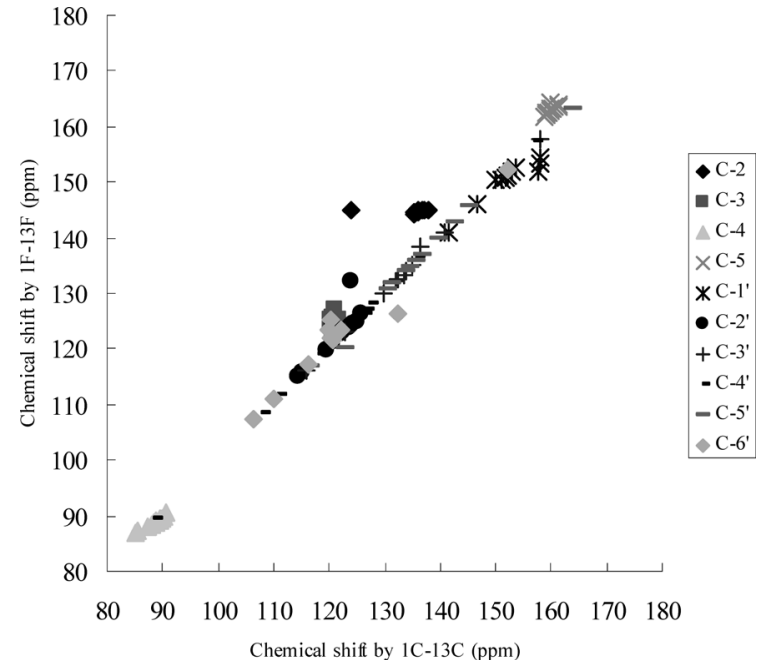

Fig. 5. Plot of Compounds $\mathbf{1 C}-\mathbf{1 3 C}$ and $\mathbf{1 F}-\mathbf{1 3 F}$ for Chemical Shifts of Carbon Generated as an Excel Worksheet Method

Each point represents chemical shifts of carbons of $\mathbf{1 C}-\mathbf{1 3 C}$ versus $\mathbf{1 F}-\mathbf{1 3 F}$.

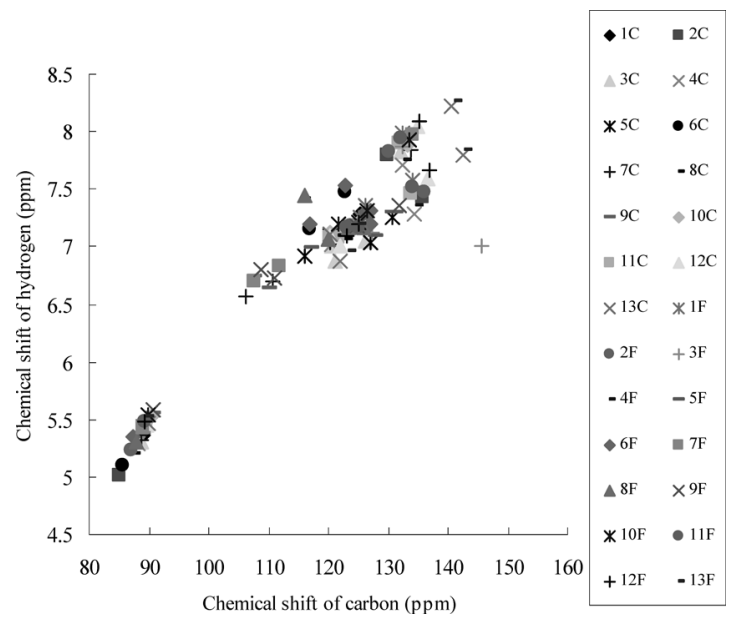

Fig. 6. Plot of Compounds $\mathbf{1 C}-\mathbf{1 3 C}$ and $\mathbf{1 F}-\mathbf{1 3 F}$ for Chemical Shifts of Carbon versus Hydrogen Generated as an Excel Worksheet Method

Each point represents chemical shifts of carbon versus hydrogen of both $\mathbf{1 C}-\mathbf{1 3 C}$ and $1 F-13 F$.

(1H, s, CHO). ${ }^{13} \mathrm{C}-\mathrm{NMR}\left(\mathrm{CDCl}_{3}\right) \delta: 52.7\left(\mathrm{COOCH}_{3}\right), 89.5$ (C-4), 89.5 (C-4'), 123.3 (C-6'), 124.7 (C-2'), 125.1 (C-3), $141.0\left(\mathrm{C}-3^{\prime}\right), 142.9\left(\mathrm{C}-5^{\prime}\right), 145.0$ (C-2), $152.6\left(\mathrm{C}-1^{\prime}\right), 162.1$ (C-5), $163.4\left(\underline{\mathrm{COOCH}}_{3}\right), 175.6(\underline{\mathrm{C} H O})$. Anal. Calcd for $\mathrm{C}_{13} \mathrm{H}_{9} \mathrm{IO}_{5}$ : C, 41.96; H, 2.44. Found: $\mathrm{C}, 41.89 ; \mathrm{H}, 2.37$.

Pattern Recognition Analysis of 5-(2'-Alkoxycarbonylsubstituted phenoxy)furfural Derivatives $(1 \mathrm{~F}-13 \mathrm{~F})$ by EI-MS and NMR Compounds $1 \mathrm{C}-13 \mathrm{C}$ and $1 \mathrm{~F}-13 \mathrm{~F}$ were pure, so the data could be easily interpreted. After constructing the EI-MS and NMR worksheet with all compounds $1 \mathrm{C}-\mathbf{1 3 C}$ and $\mathbf{1 F}-\mathbf{1 3 F}$, the results obtained are described in Figs. 4-6, which allowed each of these compounds to be classified according to the EI-MS and NMR pattern plots and their equations.

\section{Discussion}

As shown in Fig. 1, the esterification of 2-hydroxy-6methoxybenzoic acid with $\mathrm{CH}_{3} \mathrm{I}$ in the presence of $\mathrm{CsF}$ was optimized at the reaction ratio of 2-hydroxy-6-methoxyben- zoic acid $/ \mathrm{CH}_{3} \mathrm{I} / \mathrm{CsF}=1 / 1.5 / 1.5$ dissolved in DMF at room temperature for $24 \mathrm{~h}^{18)}$ According to the nucleophilic substitution reaction as shown in Fig. 2, a mixture of 5-nitrofurfural and various phenoxides in DMSO was reacted at room temperature for $23 \mathrm{~h}$ to yield compounds $\mathbf{1 F}-\mathbf{1 3 F}{ }^{20)}$

Three solvent systems, chloroform, ethyl acetate $-n$-hexane and chloroform-benzene, were applied to column chromatography for the separation of compounds $\mathbf{1 F}-\mathbf{1 3 F}$. This gentle and expeditious chromatography technique suits well solid and viscous samples. The yield and $\mathrm{mp}$ of compounds $1 \mathbf{F}-\mathbf{1 3 F}$ were from $3.1-23.6 \%$ and $50.5-100.0^{\circ} \mathrm{C}$, respectively. Some compounds (1F, 2F, 4F, 5F, 9F, 11F, 12F) were liquid and some compounds $(3 \mathrm{~F}, 6 \mathrm{~F}-\mathbf{8 F}, 10 \mathrm{~F}, 13 \mathrm{~F})$ were solid. After investigating mass spectra of compounds $\mathbf{1 F}-\mathbf{1 3 F}$, the base peaks (relative intensity $=100 \%$ ): $126 \mathrm{~m} / \mathrm{z}$ for $\mathbf{1 F}$ and $\mathbf{1 0 F}-\mathbf{1 3 F}$; $134 \mathrm{~m} / \mathrm{z}$ for $\mathbf{2 F}-\mathbf{4 F}$; $112 \mathrm{~m} / \mathrm{z}$ for $\mathbf{5 F}$; $150 \mathrm{~m} / \mathrm{z}$ for $\mathbf{6 F}$ and $\mathbf{8 F} ; 63 \mathrm{~m} / \mathrm{z}$ for $\mathbf{7 F}$ and $55 \mathrm{~m} / \mathrm{z}$ for $\mathbf{9 F}$. EIMS, UV, IR, ${ }^{1} \mathrm{H}$ - and ${ }^{13} \mathrm{C}-\mathrm{NMR}$ spectral analyses confirmed the chemical structures of compounds $1 \mathbf{F}-\mathbf{1 3 F}$. For instance, compound $\mathbf{1 F}$ was determined as $\mathrm{C}_{13} \mathrm{H}_{10} \mathrm{O}_{5}$ according to its EI-MS spectrum $\left[\mathrm{m} / \mathrm{z}\right.$ (relative intensity \%): $246\left(\mathrm{M}^{+}\right.$, 19.0)]. Its ${ }^{1} \mathrm{H}-\mathrm{NMR}$ spectrum showed two doublet signals, one singlet signal and four sets of signals with spin coupling system. The one doublet signal at $\delta 5.44(1 \mathrm{H}, \mathrm{d}, J=3.7 \mathrm{~Hz})$ was assigned to be $\mathrm{H}-4$, while the other at $\delta 7.20(1 \mathrm{H}$, d, $J=3.7 \mathrm{~Hz}$ ) was assigned to be $\mathrm{H}-3$. The singlet signal at $\delta$ $9.37(1 \mathrm{H}, \mathrm{s})$ was assigned to be the aldehyde group. The first set of spin coupling systems was observed at $\delta 7.25(1 \mathrm{H}$, d, $J=1.1 \mathrm{~Hz})$, the second set at $\delta 7.35(1 \mathrm{H}$, ddd, $J=7.7,1.1 \mathrm{~Hz})$, the third set at $\delta 7.58(1 \mathrm{H}$, ddd, $J=7.7,1.8 \mathrm{~Hz})$ and the fourth set at $\delta 7.99(1 \mathrm{H}, \mathrm{dd}, J=7.7,1.8 \mathrm{~Hz})$, attributed to the $\mathrm{H}-6^{\prime}$, $\mathrm{H}-4^{\prime}, \mathrm{H}-5^{\prime}$ and $\mathrm{H}-3^{\prime}$ of the $2^{\prime}$-methoxycarbonylphenoxy group, respectively. Furthermore, the structural assignment was also confirmed by 2D-NMR (heteronuclear multiple quantum coherence (HMQC) and heteronuclear multiple bond coherence (HMBC) correlations in Fig. 7) spectra. According to the EI-MS, UV, IR, ${ }^{1} \mathrm{H}$ - and ${ }^{13} \mathrm{C}-\mathrm{NMR}$ data, compound $1 \mathrm{~F}$ was elucidated to be 5-(2'-methoxycarbonylphenoxy)furfural.

Figure 4 shows a map of fragmented and molecular ions obtained by measuring EI-MS spectra for compounds 1C 13C and $1 F-13 F$. Each point on the map represents the fragmented and molecular ion of $\mathbf{1 C}-\mathbf{1 3 C}$ versus $\mathbf{1 F}-\mathbf{1 3 F}$. The data give an $r=0.985$ and show good performance for both $1 \mathrm{C}-\mathbf{1 3 C}$ and $1 \mathrm{~F}-\mathbf{1 3 F}$. There is an equation of $y=0.831 x+10.303$. Only two points of $11 \mathrm{C}$ versus $\mathbf{1 1 F}$ and $12 \mathrm{C}$ versus $12 \mathrm{~F}$ are clearly well separated from all the other points as a result of the halogen group. To analyze the fragmented ions of compounds $1 \mathrm{C}-13 \mathrm{C}$ and $\mathbf{1 F}-\mathbf{1 3 F}$, "active structure" is shown in Fig. 8. Figure 5 shows a chemical shift map obtained by measuring ${ }^{13} \mathrm{C}-\mathrm{NMR}$ spectra for compounds 1C-13C and 1F-13F. Each point on the map represents the chemical shift of the carbon $\left(\mathrm{C}-2,3,4,5,1^{\prime}, 2^{\prime}, 3^{\prime}, 4^{\prime}\right.$, $\left.5^{\prime}, 6^{\prime}\right)$ for $\mathbf{1 C}-\mathbf{1 3 C}$ versus $\mathbf{1 F}-\mathbf{1 3 F}$. The data give an $r=0.997$ and also show good performance for both $\mathbf{1 C}-\mathbf{1 3 C}$ and $1 \mathbf{F}-13 \mathbf{F}$. There is an equation of $y=0.995 x+0.926$. Only these C-2 points are clearly well separated from all the other points as a result of both $\mathrm{COOC}_{2} \mathrm{H}_{5}$ and $\mathrm{CHO}$ groups. Figure 6 shows a chemical shift map obtained by measuring ${ }^{1} \mathrm{H}$ - and ${ }^{13} \mathrm{C}$-NMR spectra for both $\mathbf{1 C}-\mathbf{1 3 C}$ and $\mathbf{1 F}-\mathbf{1 3 F}$. Each point on the map represents the chemical shift of the 


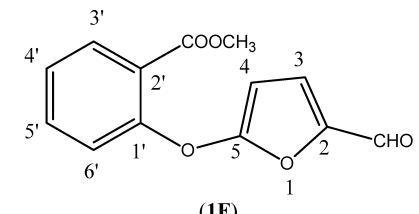

(1F)

\begin{tabular}{cccc}
\hline \hline \multicolumn{2}{c}{ HMQC correlations } & \multicolumn{2}{c}{ HMBC correlations } \\
\hline $\mathrm{H}$ & ${ }^{13} \mathrm{C}$ & ${ }^{1} \mathrm{H}$ & ${ }^{13} \mathrm{C}$ \\
\hline $\mathrm{H}-3$ & $\mathrm{C}-3$ & $\mathrm{H}-3$ & $\mathrm{C}-2, \mathrm{C}-5$ \\
\hline $\mathrm{H}-4$ & $\mathrm{C}-4$ & $\mathrm{H}-4$ & $\mathrm{C}-2, \mathrm{C}-5$ \\
\hline $\mathrm{H}-3^{\prime}$ & $\mathrm{C}-3^{\prime}$ & $\mathrm{H}-3^{\prime}$ & $\mathrm{C}-1^{\prime}, \mathrm{C}-5^{\prime}, \underline{\mathrm{COOCH}}_{3}$ \\
\hline $\mathrm{H}-4^{\prime}$ & $\mathrm{C}-4^{\prime}$ & $\mathrm{H}-4^{\prime}$ & $\mathrm{C}-2^{\prime}$ \\
\hline $\mathrm{H}-5^{\prime}$ & $\mathrm{C}-5^{\prime}$ & $\mathrm{H}-5^{\prime}$ & $\mathrm{C}-1^{\prime}, \mathrm{C}-3^{\prime}$ \\
\hline $\mathrm{H}-6^{\prime}$ & $\mathrm{C}-6^{\prime}$ & $\mathrm{H}-6^{\prime}$ & $\mathrm{C}-1^{\prime}, \mathrm{C}-2^{\prime}, \mathrm{C}-4^{\prime}$ \\
\hline $\mathrm{COOCH}$ & & & \\
\hline $\mathrm{C} \underline{H}_{3}$ & $\mathrm{COOC} \mathrm{H}_{3}$ & & \\
\hline$\underline{\mathrm{CHO}}$ & \\
\hline
\end{tabular}

Fig. 7. HMQC and HMBC Correlations of Compound $\mathbf{1 F}$

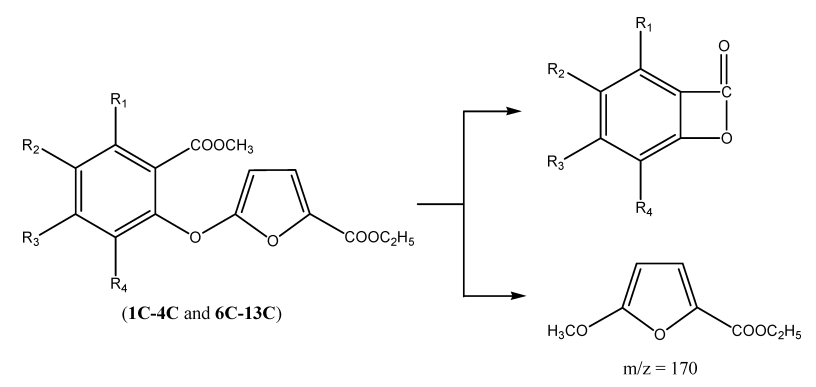

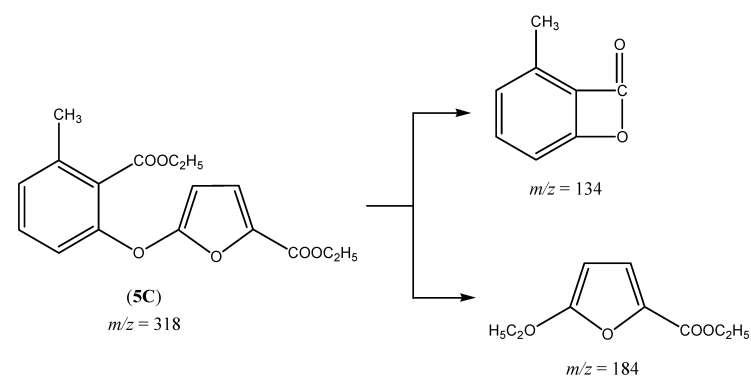

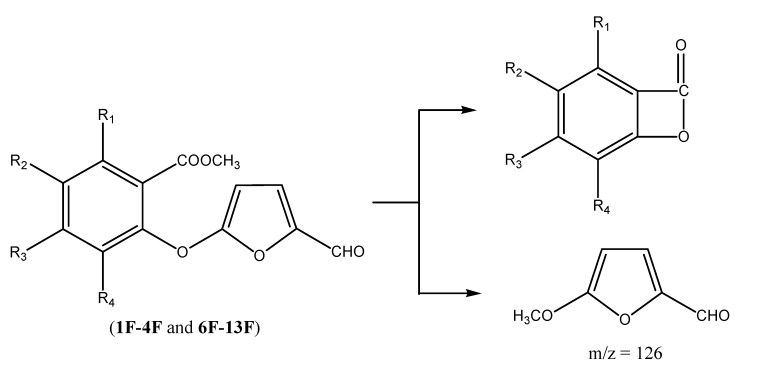

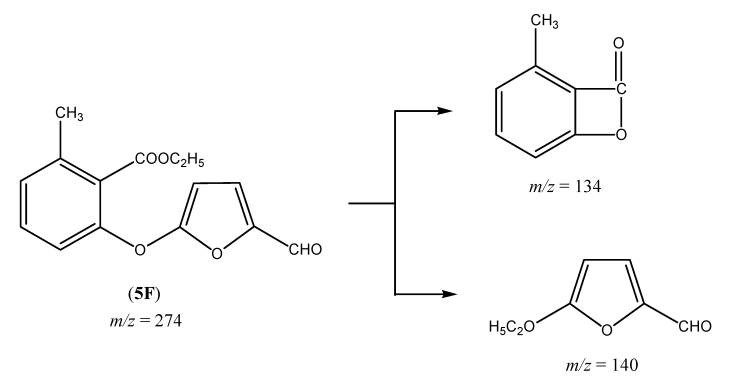

Fig. 8. Proposed EI-MS Fragmentation Patterns of Compounds 1C-13C and $\mathbf{1 F}-\mathbf{1 3 F}$

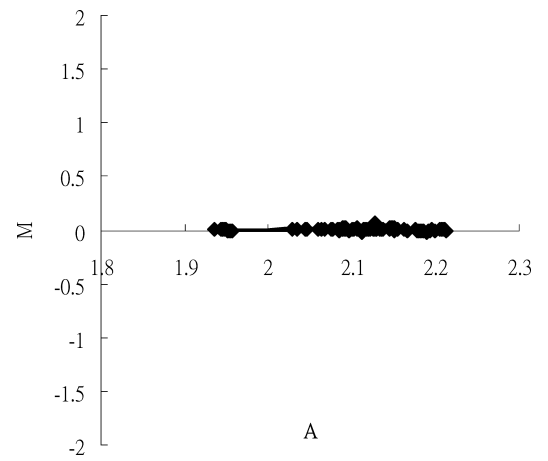

Fig. 9. The $M-A$ Plot of the Chemical Shifts of the Carbons for Both 1C-13C and $\mathbf{1 F}-\mathbf{1 3 F}$ Uses $M$ as the $y$-Axis and $A$ as the $x$-Axis

carbon versus hydrogen (C-3 vs. H-3; C-4 vs. H-4; C-3' vs. $\mathrm{H}-3^{\prime}$; C-4' vs. H-4'; C-5' vs. H-5'; C-6' vs. H-6') for both 1C-13C and 1F-13F. The data give an $r=0.868$ and also show good performance for the carbon versus hydrogen of both $\mathbf{1 C}-\mathbf{1 3 C}$ and $\mathbf{1 F}-\mathbf{1 3 F}$. There is an equation of $y=0.052 x+0.830$. These results above indicate that it unlikely that compounds $\mathbf{1 C}-\mathbf{1 3 C}$ and $\mathbf{1 F}-\mathbf{1 3 F}$ belong to more than one skeleton. In addition, these equations may be applied to analysis of new synthesized derivatives in advance. To research the probable structure skeleton of a synthesized compound, it is only necessary to add fragmented and molecular ions, and chemical shifts to the Excel worksheet and to the equation of the pattern plot on a case-bycase basis. The simple Excel worksheet analysis method will allow the combination of data from EI-MS and NMR spectrometers at different fragmented and molecular ions, and chemical shifts, respectively.

$M-A$ plots were employed to visualize differences in chemical shifts of the carbon (C-2, 3, 4, 5, 1', 2', 3', 4', 5', $\left.6^{\prime}\right)$ between both $1 \mathrm{C}-\mathbf{1 3 C}$ and $\mathbf{1 F}-\mathbf{1 3 F} . M=\log (\mathrm{chemical}$ shift of the carbon in $\mathbf{1 F}-\mathbf{1 3 F} /$ chemical shift of the carbon in $\mathbf{1 C}-\mathbf{1 3 C}$ ) represents a $\log$ ratio of the chemical shift of the carbon for both $\mathbf{1 F}-\mathbf{1 3 F}$ and $\mathbf{1 C}-\mathbf{1 3 C}$. $A=[\log (\mathrm{chemi}-$ cal shift of the carbon in $\mathbf{1 F}-\mathbf{1 3 F})+\log ($ chemical shift of the carbon in $\mathbf{1 C}-\mathbf{1 3 C}$ )]/2 is the average logarithmic chemical shift of the carbon. $M$ and $A$ were calculated for each carbon $\left(\mathrm{C}-2,3,4,5,1^{\prime}, 2^{\prime}, 3^{\prime}, 4^{\prime}, 5^{\prime}, 6^{\prime}\right)$ in both $\mathbf{1 C}-\mathbf{1 3 C}$ and $\mathbf{1 F}-\mathbf{1 3 F}$. The general assumption is that both $\mathbf{1 F}-\mathbf{1 3 F}$ and 1C-13C will not show any change in most of the chemical shifts of the carbon. Therefore, the majority of these points on the $y$-axis $(M)$ would be located at zero. In this $M-A$ plot (Fig. 9), the result fills the bill. The objective of the above analysis processes was to determine a correct skeleton of these synthesized compounds from the EI-MS and NMR patterns. The construction of this Excel worksheet will be a simple method, superior to special statistical software, for achieving EI-MS and NMR pattern recognition.

\section{Conclusion}

A series of derivatives $\mathbf{1 F}-\mathbf{1 3 F}$ had been synthesized and their structural pattern recognition had been studied by comparison with compounds $\mathbf{1 C}-\mathbf{1 3 C}$. Compounds $\mathbf{1 F}-\mathbf{1 3 F}$ were purified by column chromatography. The exact structures were elucidated and identified by EI-MS, UV, IR, ${ }^{1} \mathrm{H}-$, ${ }^{13} \mathrm{C}-\mathrm{NMR}$ and 2D-NMR (HMQC and HMBC) spectrometers. Pattern recognition analysis was studied and discussed in 
depth. According to the EI-MS pattern recognition analysis, the halogen substitute was much more changed than the methoxy and methyl substitutes. Among ${ }^{13} \mathrm{C}-\mathrm{NMR}$ pattern recognition analysis, chemical shifts of $\mathrm{C}-2$ showed greater differences because of the difference between $\mathrm{COOC}_{2} \mathrm{H}_{5}$ and $\mathrm{CHO}$ groups. However, chemical shifts of ${ }^{13} \mathrm{C}-\mathrm{NMR}$ versus ${ }^{1} \mathrm{H}-\mathrm{NMR}$ showed no obvious differences in the pattern recognition analysis. The $M-A$ plot also shows good performance. The simple Excel worksheet analysis method requires no prior knowledge of the data and the results are statistically valid. The spectroscopic data mining technique thus offers high-throughput screening for pattern recognition analysis and structural skeleton identification. It is possible to determine the correct skeleton of synthetic structures in future structural elucidation studies. Mass and NMR spectral data are essential to identify the compounds $\mathbf{1 C}-\mathbf{1 3 C}$ and $\mathbf{1 F}$ 13F. If these data work as an index of the biological activities, it is very useful to avoid some of complicated procedures. For the preliminary tests of the biological activities, compound 1F also showed anti-platelet aggregation, anti-allergic and anti-inflammatory activities. The study of antiplatelet aggregation, anti-allergic and anti-inflammatory ac-

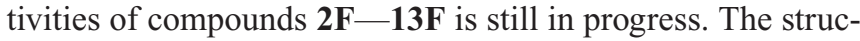
ture-activity relationship will be established, with results to be reported in future.

Acknowledgements The author wishes to express his gratitude to Dr. Chin-Sheng Wu for the scientific support. This work was financially supported by the China Medical University, Taichung, Taiwan, R.O.C.

\section{References}

1) Chang C. L., Wu C. S., Teng C. M., J. Chin. Med. Sci., 3, 1-8 (2002).
2) Chang C. L., Wu C. S., Wang S. W., Wang J. P., Chin. Pharm. J., 54, 95-113 (2002).

3) Borawski J., Rydzewski A., Pawlak K., Azzadin A., Buczko W., Mysliwiec M., Thromb. Res., 90, 171-180 (1998).

4) Hiramatsu N., Kageyama K., Acta Anaesthesiol. Scand., 47, 53-57 (2003).

5) Hogan D. F., Andrews D. A., Talbott K. K., Green H. W., Ward M. P., Calloway B. M., Am. J. Vet. Res., 65, 327-332 (2004).

6) Wertheimer R., Blessmann G., Klin. Monatsbl. Augenheilkd., 210, 93-96 (1997).

7) Simons F. E., Roberts J. R., Gu X., Kapur S., Simons K. J., J. Allergy Clin. Immunol., 103, 223-226 (1999).

8) Rudolph K., Bice D. E., Hey J. A., McLeod R. L., Am. J. Rhinol., 17, 227-232 (2003).

9) Auer M., Brezinka C., Eller P., Luze K., Schweigmann U., Schwarzler P., Ultrasound Obstet. Gynecol., 23, 513-516 (2004).

10) Yamamoto T., Matsuura K., Shintani S., Hara A., Miyabe Y., Sugiyama T., Katagiri Y., Biol. Pharm. Bull., 21, 1148-1153 (1998).

11) Karademir S., Oguz D., Senocak F., Ocal B., Karakurt C., Cabuk F., Pediatr. Int., 45, 676-679 (2003).

12) Praisler M., Dirinck I., Van Bocxlaer J., De Leenheer A., Massart D. L., Talanta, 53, 177-193 (2000).

13) Hohenner M., Wachsmuth S., Sagerer G., Knowledge-Based Systems, 18, 207-215 (2005).

14) Liu J., Sun J., Wang S., International Journal of Computer Science and Network Security, 6, 57-61 (2006).

15) Piao X. L., Park J. H., Cui J., Kim D. H., Yoo H. H., J. Pharm. Biomed. Anal., 44, 1163-1167 (2007).

16) Shum K. C., Chen F., Li S. L., Wang J., But P. P. H., Shaw P. C., J. Sep. Sci., 30, 3233-3239 (2007).

17) Aursand M., Standal I. B., Axelson D. E., J. Agric. Food Chem., 55, $38-47$ (2007).

18) Sato T., Otera J., Nozaki H., J. Org. Chem., 57, 2166-2169 (1992)

19) Tanaka A., Usui T., Shimadzu M., J. Heterocycl. Chem., 18, 12411244 (1981).

20) Tanaka A., Usui T., Shimadzu M., Chem. Pharm. Bull., 28, 2846 2849 (1980). 